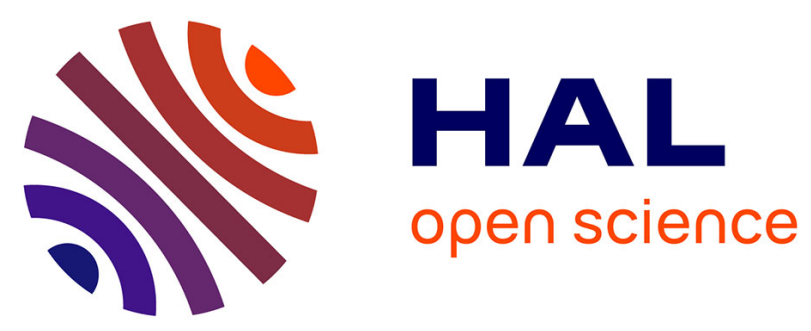

\title{
Interpretations and performances of linear reception in downlink TD-CDMA and multi-sensor extension
}

Laurent Ros, Geneviève Jourdain, Marylin Arndt

\section{To cite this version:}

Laurent Ros, Geneviève Jourdain, Marylin Arndt. Interpretations and performances of linear reception in downlink TD-CDMA and multi-sensor extension. Annals of Telecommunications - annales des télécommunications, 2001, 56 (5-6), pp.275-290. hal-00275644

\section{HAL Id: hal-00275644 \\ https://hal.science/hal-00275644}

Submitted on 24 Apr 2008

HAL is a multi-disciplinary open access archive for the deposit and dissemination of scientific research documents, whether they are published or not. The documents may come from teaching and research institutions in France or abroad, or from public or private research centers.
L'archive ouverte pluridisciplinaire HAL, est destinée au dépôt et à la diffusion de documents scientifiques de niveau recherche, publiés ou non, émanant des établissements d'enseignement et de recherche français ou étrangers, des laboratoires publics ou privés. 


\title{
Interpretations and performances of linear reception in downlink TD-CDMA and multi-sensor extension*
}

\author{
Laurent ROS ** \\ Geneviève JOURDAIN ** \\ Marylin ARNDT ***
}

\begin{abstract}
This paper deals with a TD-CDMA multi-user system downlink through a radio-mobile multipath channel, such as TDD-UMTS. We propose interpretations and equivalent models specific to such a multiple access mode, mostly via a frequency approach. The expressions and performances of the classic linear multi-user detectors are then reformulated. Furthermore, performance computation is applied to indoor and vehicular channel environments, showing the benefit of joint detection and diversity reception with two or three sensors at the mobile.
\end{abstract}

Key words : Mobile radiocommunication downlink, Code division multiple access (CDMA), Multi-sensor antenna, Multi-path and multi-user channel, Joint detection, Aliased cross-channels spectral matrix, TDD-UMTS.

\section{INTERPRÉTATIONS ET PERFORMANCES DU RÉCEPTEUR LINÉAIRE EN LIEN DESCENDANT TD-CDMA ET EXTENSION MULTI-CAPTEUR}

\section{Résumé}

Cet article s'intéresse au lien descendant d'un système radio-mobile multi-utilisateur TD-CDMA soumis au multi-trajet, tel le mode TDD de l'UMTS. Il propose des interprétations et des modèles équivalents spécifiques à ce mode d'accès multiple, avec une approche le plus souvent fréquentielle. Les expressions et performances des récepteurs linéaires classiques sont alors reformulées. A titre d'illustration, le calcul de performances est appliqué aux modèles d'environnement "Indoor" et "Vehicular", montrant le bénéfice de la détection conjointe ainsi que l'apport d'une réception avec 2 ou 3 capteurs sur le mobile.
Mots clés : Radiocommunication mobile lien descendant, Accès multiple à répartition par codes, Antenne multicapteur, Canal multi-trajet et multi-utilisateur, Détection conjointe, Matrice spectrale des inter-canaux repliés, TDD-UMTS.

\section{Contents}

I. Introduction.

II. Multi-user transmission model.

III. Structure of the joint linear detector.

$I V$. Interpretations and models of CDMA.

$V$. Expressions and performances of linear detectors.

VI. Extension to multi-sensor reception.

VII. Average performances in Indoor and Vehicular environments.

VIII. Conclusion.

References (21 ref.)

\section{INTRODUCTION}

The normalization group $3 \mathrm{GPP}$ is preparing the standards for the third generation of radio-mobile system, UMTs (Universal Mobile Telecommunications System). Among them, the TDD (Time Division Duplex) mode [21] permits, for one or several users, the simultaneous transmission, during each TDMA slot, of spread QPSK symbols with different CDMA signatures. The uplink and downlink work at the same carrier frequency around $2 \mathrm{GHz}$, with quadrature modulation by two 3.84 Mchip/s-binary streams of 5 $\mathrm{MHz}$-spectral width. A synchronization channel and a midamble, transmitted at the slot rate, make the synchronization and channel estimation easier, independently of symbol estimation mainly considered here. The spread spectrum systems were initially introduced in single-user situation in order to ensure discretion or to fight against undesired channel effects [2]. They were calibrated, as far as possible, so that the symbol time was superior to the temporal spread of the channel, in such a way that the receiver was simply based on a matched filtering ( $\mathrm{MF}$ ).

\footnotetext{
* This work was supported by France Telecom R\&D, from a CTI convention between FT R\&D and LIS (INPG/UJF/CNRS)

** Laboratoire des Images et des Signaux (LIS), domaine universitaire, BP46, 38402 Saint-Martin d'Hères, France

*** France-Telecom R\&D (DIH/OCF), 28 chemin du vieux chêne, BP98, 38243 Meylan, France
} 
Therefore, the transmission was robust but featured a very bad spectral efficiency.

In contrast, the systems which are considered at today should provide an increase in the network global capacity. So, the spread messages from different users are superimposed ${ }^{1}$, in a number inferior but as close as possible as the spreading factor; this results in a non negligible part of Multiple Access Interference (MAI). Moreover, the channel spreading may largely go beyond the symbol time in vehicular environment [20], leading to a substantial Inter-Symbol Interference (ISI). To compensate interference and better detect each user's symbol, the linear joint detection with temporal depth is a receiving strategy which uses the knowledge of the codes of other users, whereas the simple matched filter considers the MAI and ISI just as wideband noise. The linear joint detection is not optimal for minimizing the Bit Error Rate (BER) but ensures a good compromise between performances and complexity, especially in TDD mode where the codes are short (16-chip long maximum). Nevertheless, the interference cancelling, complete or partial, leads to an amplification of thermal additive noise. This effect can be largely reduced by a multisensor coherent processing on the mobile, which improves the waveform orthogonality and facilitates the system inversion. We only consider non adaptive antenna for the base station transmitter.

The objectives of this paper are:

(A) to interpret the CDMA system in downlink (in section IV), usually with a frequency approach, natural in spread spectrum systems. We will establish the wide-band equivalent model, leading to an analogy with the source separation in antenna processing, and the symbol-band equivalent model which is identical to the one without spreading systems,

(B) to make a synthesis on the theoretical structures and performances of "classical" linear receivers for selective channels (in sections III, V, VI), established from models described in (A). An exhaustive synthesis has been carried out by the authors of [4] but from block temporal formulation using a transfer matrix of all the symbols in the slot. The frequency approach permits here to simplify the model and to take naturally into account the selective channels,

(C) to evaluate the average performances in Vehicular and Indoor environment models (in section VII) by application of the expressions of (B) in order to measure the interest of joint detection and multi-sensor reception over selective and non selective channels.

\footnotetext{
${ }^{1}$ To simplify the description we abusively associate, in the whole article, one active code to one user. In real applications, several codes may be affected to the same user in order to increase its information rate (multi-code situation).
}

\section{MULTI-USER TRANSMISSION MODEL}

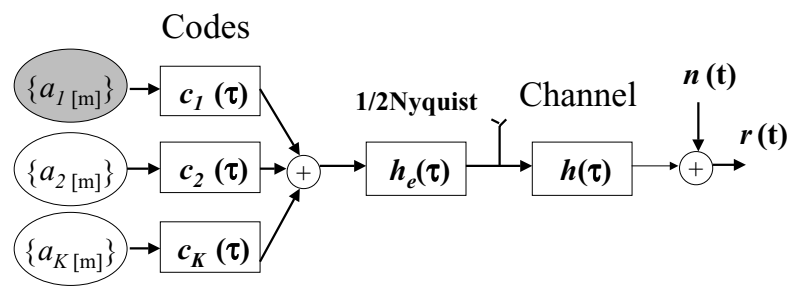

Fig. 1.- Baseband model of transmission. Modèle en bande de base de la transmission.

The continuous-time baseband representation (complex envelope) of the received signal, observed during one time slot (containing $M$ symbols), is modeled as:

$$
r(t)=T s . \sum_{k=1}^{K} \sum_{m=1}^{M} a_{k[m]} . g_{k}(t-m T s)+n(t)
$$

where

- $a_{k[.]}$ are the QPSK symbols transmitted by the $k$-th source at the "symbol times" $m T s$,

- $g_{k}=c_{k} * h_{e} * h$ is the overall wide-band waveform of the $k$-th user. It results from the convolution between the $k$-th spreading code, the half Nyquist (squareroot raised cosine filter "RRC" with roll-off $\left.r_{o f f}\right)$, and the channel,

- $n(t)$ is a baseband additive complex noise.

We consider the following context of downlink multi-user communication:

- the number of users (or active codes) $K$ is less or equal to the spreading factor $Q$;

- the $K$ active codes (taken among a set of $Q$ known codes) are supposed known at the receiver, which is not necessarily the common situation (necessity of a control channel);

- the codes of different users are made from $Q$ binary complex entity named "chips". The codes are linearly independent and orthogonal at zero delay (algebraically meaning), which is expressed by:

$$
\gamma_{i k}^{c o d e}[0]=\sum_{q=0}^{Q-1} c_{i[q]} c_{k[q]}^{*}=\delta_{i k}
$$

where $\delta$. is the Kronecker symbol whereas $\delta($.$) is$ the Dirac distribution. The impulse response of one code lasts for $T s$, and is defined for the $k$-th user by: $c_{k}(\tau)=\sum_{q=0}^{Q-1} c_{k[q]} \delta(\tau-q T c)$, where $T c=T s / Q$ is the chip duration;

- the transmitted symbols are stationary, with zero mean and power $A^{2}$; they are uncorrelated temporally and from one user to another, and also uncorrelated with the additive noise;

- "downlink" with "non intelligent Base Station": all $K$ user's signals appear at each mobile sensor after transmission over the same propagation channel and 
with the same power. The channel $h$ is supposed to be deterministic, time-invariant during the observation (1 slot), linear and causal with a temporal spread limited to $W_{c}$ chips. The structures studied at the sequel need only these assumptions for the channel. Nevertheless in radio-mobile communications, the channel is generally constituted by multiple paths, and its impulse response is modeled by:

$$
h(\tau)=\sum_{i=1}^{L_{t}} \alpha_{i} . \delta\left(\tau-\tau_{i}\right)
$$

where $L_{t}$ is the number of paths, $\alpha_{i}$ are the complex amplitudes and $\tau_{i}$ the propagation delays of different paths, with $\tau_{i} \leq W_{c} . T c, \forall i \in\left\{1, . ., L_{t}\right\}$;

- the complex noise is an additive white Gaussian noise, with two-sided power spectral density $2 N_{0}$ (corresponding to a two-sided psd $\frac{N_{0}}{2}$ for the real noise on carried band at the entrance of the receiver);

- two slots of symbols are isolated by a guard time above $W_{c} . T c$;

- we suppose perfect timing recovery (using the synchronization channel) and also perfect channel estimation (using the midamble).

We suppose by convention only one desired code (or desired user), the code number one.

We define the average energy per bit at the entrance of the receiver (on carried band) for the desired user: $E_{b}=\frac{1}{4} A^{2} T s \gamma_{11[0]}$, where $\frac{\gamma_{11[0]}}{T s}$ is the zero-delay auto-correlation of the waveform $g_{1}(\tau)$ of user " 1 ".

Note: the assumption of no variation of the channel during the slot $\left(T_{\text {slot }}=667 \mu \mathrm{s}\right)$ is fully justified for a mobile moving with a speed $v_{m}$ of $3 \mathrm{~km} / \mathrm{h}$. Indeed, for a carrier radio-wave (celerity $v_{0}$ ) around $f_{0}=2 G H z$, the Doppler spread is defined by $\Delta f_{d}=$ $\frac{v_{m}}{v_{0}} \cdot f_{0}$ and the channel coherence time $T_{c o h}=\Delta f_{d}^{-1}$ is around $200 \mathrm{~ms}$. The hypothesis limit occurs with a vehicular moving at $v_{m}=120 \mathrm{~km} / \mathrm{h}\left(T_{\text {coh }} \approx 5 \mathrm{~ms}\right)$ since the complex amplitudes of the paths may suffer from phase rotation up to $\frac{\frac{1}{2} T_{\text {slot }}}{T_{\text {coh }}} .360^{\circ}$ around $+/-$ $25^{\circ}$ during one $1 / 2$ slot, in the case of deterministic Doppler model. We might have interest to updapte the knowledge of the complex amplitudes several times in the slot for optimizing the symbol detection. On the other hand, since the mobile speed $v_{m}$ is always negligible compared to the wave celerity $v_{0}$, the variation of the path delay $\frac{v_{m}}{v_{0}} \cdot T_{\text {slot }}$ is negligible during the slot (around $10^{-4} T_{c}$ ).

\section{STRUCTURE OF THE JOINT LINEAR DETECTOR}

With infinite response, the symbol by symbol linear detector [17] can take the shape of an imposed structure with one matched filter bank followed by a bank of discrete equalizing filters working at symbol rate,

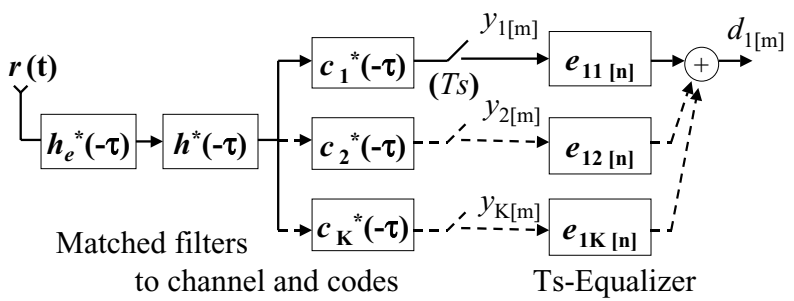

Fig. 2.- Joint linear detector (with $\gamma_{11[0]}=1$ ). Récepteur linéaire conjoint (avec $\left.\gamma_{11[0]}=1\right)$.

as seen in figure 2. It is easy to verify this, under classical criteria (Zero-Forcing, MMSE).

\section{III.1. Channel- and code-matched filter bank}

The receiver front-end applies to $r(t)$ a bank of continuous filters $g_{k}{ }^{H}, k=1 \ldots K$ matched to each global waveform, made by the chip pulse (RRC), the channel response and each of the $K$ codes. By convention, the exponent $(.)^{H}$ represents hermitian transform for a function $h\left(\right.$ i.e. $h^{H}(\tau)=h^{*}(-\tau)$ : matched filter) just as the hermitian transposition for vectors or matrix. The $K$ outputs of filters $g_{k}{ }^{H}$, sampled synchronously at symbol time, constitute a sufficient statistic of the continuous received signal $r(t)$ for the symbol estimation $[1,16]$ :

$$
y_{k[m]}=\left(g_{k}{ }^{H} * r\right)(t)_{\mid t=m T s}
$$

Thus, the knowledge of $\left\{y_{k[m]}\right\}, k=1 \ldots K, m=1 \ldots M_{+}$ is sufficient to know the likelihood function of received signal and to carry out the optimal processing which minimizes the error probability (or BER). For the treatment of one slot, we must consider a number $M_{+}$of samples slightly greatest than the effective number $M$ of symbols because of the channel temporal spread and because of the matched filter. In this article, we will not consider the optimal theoretical post-processing, i.e. the Maximum Likelihood Sequence Estimator, but only the detectors which treat linearly the $\left\{y_{k[m]}\right\}$ and make the decisions symbol by symbol. The $T s$-sampled outputs $\left\{y_{k[m]}\right\}$ are expressed from discrete time convolutions of the transmitted symbols, disturbed by an additive noise:

$$
y_{k[m]}=\sum_{i=1}^{K}\left(\gamma_{i k} * a_{i}\right)_{[m]}+\eta_{k[m]}
$$

where $\gamma_{i k[n]}$ is the sampling, at symbol time, of the continuous and deterministic cross-correlation function $\gamma_{i k}^{\text {cont }}(\tau)=\left(g_{i} * g_{k}{ }^{H}\right)(\tau)$ between the wide-band waveforms of users $i$ and $k$ :

$$
\gamma_{i k[n]}=T s \cdot \gamma_{i k}^{c o n t}(\tau)_{\mid \tau=n T s}
$$

After matched filtering and synchronous sampling, the equation (5) shows that all the system can be 
modeled at discrete time $T s$. The series $\left\{\gamma_{i k[n]}\right\}, \forall n \in$ $\mathbb{Z}$, providing the discrete filtering, has no dimension. Through a multi-path channel, the waveforms are generally not orthogonal and some ISI and MAI interference occurs with respectively secondary terms $\gamma_{11}$ at non zero delays and cross-channel terms $\gamma_{1 i}, i \neq 1$ at the different delays:

$$
\begin{gathered}
y_{1[m]}=\underbrace{\gamma_{11[0]} \cdot a_{1[m]}}_{\text {desired part }}+\underbrace{\sum_{n \neq m} \gamma_{11[m-n]} \cdot a_{1[n]}}_{I S I}+ \\
\underbrace{\sum_{i=2}^{K} \sum_{n} \gamma_{i 1[m-n]} \cdot a_{i[n]}}_{\text {MAI }}+\underbrace{\eta_{1[m]}}_{\text {Noise }}
\end{gathered}
$$

The necessity and the difficulty of the equalization will be entirely conditioned by the values $\left\{\gamma_{i k}[n]\right\}$. Recalling that:

* In the case of a single-user system or without joint detection, only the first branch is used, operating a coherent combination relative to the different paths, called Rake; $y_{1[\mathrm{~m}]}$ may then be the decision variable. * In the case of a multi-user system but a singlepath channel, the duration of the code limited to $T s$ (and the respect of the Nyquist criterion at the chip time for $h_{e}$ ) leads to no interference at non zero delay since $\gamma_{i k[n]}=0, \forall n \neq 0$. Moreover, when the codes are orthogonal, there is no interference at the zero delay since $\gamma_{i k[0]}=\delta_{i k}$ : only the first branch of the MF bank is useful, reduced to the receiving filter RRC followed by a correlation with the desired code.

* In the case of a multi-path channel with weak temporal spread relative to symbol time, the contribution at non zero symbol time delays are negligible and only the MAI at the zero delay may be considered. It is similar to a situation where the waveforms would last $T s$, but without being orthogonal: this is the case studied by most authors $[16,6,5]$, corresponding to a well calibrated system, but it leaves out a number of situations in a vehicular environment with the TDD-UMTS parameters.

\section{III.2. Bank of discrete equalizers $(T s)$}

In order to form a decision variable $d_{1[\mathrm{~m}]}$ used to estimate the user's one QPSK symbols $a_{1[\mathrm{~m}]}$ by quadrant detection, an equalizer linearly combines the outputs $\left\{y_{k[m]}\right\}$ of the $K$ branches of the matched filtersampler bank, after passing them through a bank of discrete filters $\left\{e_{1 k[n]}\right\}$ working at symbol time:

$$
d_{1[m]}=\frac{1}{\gamma_{11[0]}} \cdot \sum_{k=1}^{K}\left(e_{1 k} * y_{k}\right)_{[m]}
$$

Having described the structure of the detector, we will now derive and interpret equivalent models of the CDMA system before setting the performances and expressions of the multi-user equalizer.

\section{INTERPRETATIONS AND MODELS OF CDMA}

During transmission, the equation (1) expresses the multiple access based on orthogonal waveforms (symbol pulse-shapes) from one user to another, for an ideal channel. For the particular CDMA case, each pulse $g_{k}$, for $k=1$ to $K$, occupies all the available time-frequency plane $\left(T s \cdot \frac{Q}{T s}\right)$ instead of being located in time (width $\frac{T s}{K}$ in TDMA) or in frequency (width $\frac{1}{T s}$ in FDMA). The number $\left(\frac{Q-K}{Q}\right)$ plays the role of a code margin with regard to the system saturation, same as the margins provided by the guard times or the guard bands in TDMA or FDMA.

By introducing in (10) the impulsive form $a_{k}(t)$ of the different user symbols, the received signal defined in (1) can be expressed classically as a time continuous convolution between the symbols and the global wide-band waveforms, disturbed by an additive noise:

$$
\begin{aligned}
r(t) & =\sum_{k=1}^{K}\left(g_{k} * a_{k}\right)(t)+n(t) \\
\text { with } \quad a_{k}(t) & =T s \cdot \sum_{m=1}^{M} a_{k[m]} . \delta(t-m T s)
\end{aligned}
$$

This expression allows describing the chain as a cascade of linear filters excited by the impulsive symbols streams, exactly the same way as a linear modulation without spreading. The difference here is that the pulse shape is wide-band compared to the symbol band of width $\frac{1}{T s}$. This formulation will facilitate the frequency transformation in the following paragraph.

\section{IV.1. Interpretation at transmission (multi-band transmission)}

The result of a single observation during the slot can be analysed in the frequency domain with the Fourier transform. The signals $a(t)$ and $n(t)$ are here considered as deterministic (being particular occurences during a slot of random variables), which avoids to treat with (cyclic) autocorrelation functions of the received signal. The Fourier Transform of equation (9) gives:

$$
\hat{r}\left(f_{w}\right)=\sum_{k=1}^{K} \hat{g}_{k}\left(f_{w}\right) \cdot \hat{a}_{k}\left(f_{w}\right)+\hat{n}\left(f_{w}\right)
$$

where by convention:

$-f_{w}$ is used for any frequency while $f$ is a frequency of the symbol-band, included in $\left[-\frac{1}{2 T s},+\frac{1}{2 T s}[\right.$. So, for all $f_{w}$, there is a single frequency $f$ and a single relative integer $q$ such that $f_{w}=f+\frac{q}{T s}$,

- the continuous Fourier transform of a function $h$ in continuous time or discrete time (then obtained via Z transform) is noted $\hat{h}($.). 
The transmission bandwidth, defined by the support of $\hat{g}_{k}\left(f_{w}\right)$ is limited to the band of the half Nyquist filter, that is $Q_{r} \frac{1}{T s}$ where $Q_{r}$ is the integer part of $\left(1+r_{o f f}\right)$. $Q$, expressing the filter bandwidth excess. With the UMTs parameters $Q=16$ and $r_{o f f}=0.22, Q_{r}$ is odd and equal to 19 .

The $Q_{r}$ symbol bands (of width $\frac{1}{T s}$ ) which compose the wide-band are then centred around the frequencies $f_{w}=-\frac{Q_{r / 2}}{T s}, \ldots, 0, \ldots,+\frac{Q_{r / 2}}{T s}$ with the definition of $Q_{r / 2}$ such that $Q_{r}=2 Q_{r / 2}+1$.

Note: Taking into account the RRC attenuation, the portions not included in this $Q_{r}$ bands are ignored. If one does not want to lose any information, we might have taken $Q_{r}+1=20$ instead of $Q_{r}$ and choose for $f$ a range equal to $\left[0,+\frac{1}{T s}[\right.$.

Because of the characteristic of "sampling" (Cf (10)) of $a_{k}(t)$ at the symbol rate (or the cyclostationarity of the digital communication signals), $\hat{a}_{k}\left(f_{w}\right)$ is periodic with a period of $\frac{1}{T s}$ :

$$
\hat{a}_{k}(f)=\hat{a}_{k}\left(f+\frac{q}{T s}\right), \forall q \in \mathbb{Z}
$$

The expression (11) is then written inside the useful wide-band:

$$
\hat{r}\left(f+\frac{q}{T s}\right)=\sum_{k=1}^{K} \hat{r}_{k}\left(f+\frac{q}{T s}\right)+\hat{n}\left(f+\frac{q}{T s}\right)
$$

with $\forall q=-Q_{r / 2}, \ldots,+Q_{r / 2}$ :

$$
\hat{r}_{k}\left(f+\frac{q}{T s}\right)=\hat{g}_{k}\left(f+\frac{q}{T s}\right) \cdot \hat{a}_{k}(f)
$$

The equation (14) expresses the frequency diversity of order $Q_{r}$ inherent to spread spectrum systems: for a given user " $k$ ", the same binary message $a_{k[m]}, m=1 \ldots M$, with Fourier transform $\hat{a}_{k}(f)$ on the complete symbol band, is transmitted in $Q_{r}$ different symbol-bands. Generally (with weak excess bandwidth or roll-off), the portions outside the chipband (width $\frac{1}{T c}$ ) are attenuated and only a diversity of order $Q$ is considered, according to the temporal structure of codes, measured at the chip rate.

In an equivalent way, emphasis can be put on diversity by means of a multi-carrier modulation concept. From the periodicity of $\hat{a}_{k}(f)$ and the impulsive form of the code $c_{k}(\tau)$ with finite length $T s$, the $k$ th user's signal, before RRC and channel filtering, is expressed in frequency domain by:

$$
\begin{gathered}
\hat{e}_{k}\left(f_{w}\right)=\left(\hat{c}_{k}^{\text {per }} * \hat{a}_{k}^{\text {rect }}\right)\left(f_{w}\right) \\
\text { with: } \hat{c}_{k}^{\text {per }}\left(f_{w}\right)=\sum_{n} \sum_{q=-\frac{Q}{2}}^{+\frac{Q}{2}-1} \hat{c}_{k}\left(\frac{q}{T s}\right) \cdot \delta\left(f_{w}-\frac{q}{T s}-\frac{n}{T c}\right) \\
\hat{a}_{k}^{\text {rect }}\left(f_{w}\right)=\hat{a}_{k}\left(f_{w}\right) \cdot \operatorname{sinc}\left(\pi T s f_{w}\right)
\end{gathered}
$$

Unlike OFDM (Orthogonal Frequency Division Multiplexing), for a given user, the sub-carriers are modulated by the same information (complex binary message). There are spaced by $\frac{1}{T s}$ and have complex amplitudes $\hat{c}_{k}\left(\frac{q}{T s}\right)$ given by the DFT (Discrete Fourier Transform) of the $Q$ chips of the code. The messages of different users will easily be separated at the receiver, at least over ideal channel, thanks to the codes orthogonality (independency) property, which is expressed in the frequency-domain by the linear orthogonality (independency) of the different sets of $Q$ sub-carriers $\left\{\hat{c}_{k}\left(\frac{q}{T s}\right)\right\}$.

Note: we can establish the equation (15) more naturally by representing the spread-spectrum as the result of the multiplication of the continuous symbol stream $a_{k}^{r e c t}(t)$ (shaped into a rectangular pulse of length $T s$ ) by the periodic version of the code $c_{k}^{\text {per }}(t)$ (with period $T s$ ). The expression of the periodic code in Fourier series gives then directly the multi-carrier modulation scheme. This other equivalent representation of the spreading system, i.e. the "multiplicative representation", is closer to the design, and probably more ancient [3]. However, it is more difficult to manipulate theoretically than the representation with linear filters, generally adopted.

\section{IV.2. Interpretation at reception}

Assuming a perfect knowledge of the channel and timing synchronization, the outputs of the bank of MF-samplers can be synthesized from a linear transformation of the transmitted symbols, without mentioning the wide-band received signal. The temporal expression, at discrete time $T s$, of the "symbol-band model" has been established in (5).

The transfer $\hat{\gamma}_{i k}(f)$ between the symbols of the source " $i$ " and the output of the branch " $k$ " is expressed in frequency-domain by Fourier transform of equation (6) as the aliasing of the wide-band cross-spectrum $\hat{\gamma}_{i k}^{\text {cont }}\left(f_{w}\right)$ in the symbol-band:

$$
\hat{\gamma}_{i k}(f)=\sum_{q=-Q_{r / 2}}^{+Q_{r / 2}} \hat{\gamma}_{i k}^{c o n t}\left(f-\frac{q}{T s}\right)
$$

with:

$$
\begin{aligned}
\hat{\gamma}_{i k}^{c o n t}\left(f_{w}\right) & =\hat{g}_{i} \hat{g}_{k}^{*}\left(f_{w}\right) \\
& =|\hat{h}|^{2}\left|\hat{h}_{e}\right|^{2} \hat{c}_{i} \hat{c}_{k}^{*}\left(f_{w}\right)
\end{aligned}
$$

We can now explain the notions of aliased equivalent channels in the particular case of CDMA.

\section{aliased Tx/Rx Global Channels}

At the first branch output, the transfer $\hat{\gamma}_{11}(f)$ is equivalent to an aliased Trans-Receiving Global Channel (aT/RGC) in the symbol band for the desired user, the transfer $\hat{\gamma}_{i 1}(f)$ to an aT/RGC for the interfering 
user " $i \neq 1 "$. Expressed in frequency, the absence of ISI on the branch " 1 " corresponds to an aT/RGC white for the desired user, and the absence of MAI corresponds to the nullity of the aT/RGC for all the interfering users. These properties are absolutely false for the T/RGC before aliasing, $\hat{\gamma}_{i k}^{\text {cont }}\left(f_{w}\right)$, because of inter-chip and inter sub-chip interference on the wide-band channels outputs before sampling. It simply expresses, for the desired user, a coherent recombining of the sub-carriers and for the interfering users, a destructive re-combining. With a single-path channel, the orthogonality (2) and the finite length of the codes ensures these conditions. It is easy to show with the Poisson formula that $\forall f \in\left[-\frac{1}{2 T s},+\frac{1}{2 T s}[\right.$ :

$$
\sum_{q=-\frac{Q}{2}}^{+\frac{Q}{2}-1}\left(\hat{c}_{k} \cdot \hat{c}_{j}^{*}\right)\left(f-\frac{q}{T s}\right)=\gamma_{k j}^{c o d e}[0]=\delta_{k j}
$$

The respect of the first Nyquist criterion, relative to the chip time $T c$ for $h_{e} * h_{e}{ }^{H}$, leads then to:

$$
\sum_{q=-Q_{r / 2}}^{+Q_{r / 2}}\left(\hat{c}_{k} \cdot\left|\hat{h}_{e}\right|^{2} \cdot \hat{c}_{j}^{*}\right)\left(f-\frac{q}{T s}\right)=\delta_{k j}
$$

For the desired user, the equation (19) simply expresses the first Nyquist criterion relative to the symbol time $T s$ for $g_{1} * g_{1}{ }^{H}$ with an ideal channel. When the channel is not single-path, the aliasing equation (16) explains the robustness of the spread-spectrum to selective channels: the aT/RGC seen by the desired user symbols is finally a channel with width $\frac{1}{T s}$ obtained from the coherent superposition of $Q_{r}$ channels with same width, averaging the selectivity imposed to the different bands.

Figure 3 shows the $\mathrm{T} / \mathrm{RGC}$ and aT/RGC on the branch one for the desired user and for the interferer "2", with a channel taken from Vehicular B model, characterized by a temporal spreading around $5 \mathrm{Ts}$. The power of Interference and Noise (IN) at the first branch output is derived from the temporal expression of the ISI, MAI and Noise terms defined in (7):

$$
\begin{gathered}
(I N) \triangleq E\left\{\left|y_{1[m]}-\gamma_{11[0]} \cdot a_{1[m]}\right|^{2}\right\} \\
=A^{2} \sum_{n \neq 0}\left|\gamma_{11[n]}\right|^{2}+A^{2} \sum_{k=2}^{K} \sum_{n}\left|\gamma_{k 1[n]}\right|^{2}+2 N_{0} \cdot \frac{\gamma_{11[0]}}{T s}
\end{gathered}
$$

The expression of (IN) vs frequency, established with the Parseval relation applied to equation (20), shows that the Interferference and Noise power depends only on the form of the aT/RGC :

$$
\begin{aligned}
& (I N)=\mathrm{A}^{2} \mathrm{Ts} \int_{\frac{1}{T s}}\left|\hat{\gamma}_{11}(f)-\gamma_{11[0]}\right|^{2} d f \\
& +\mathrm{A}^{2} \mathrm{Ts} \sum_{k=2}^{K} \int_{\frac{1}{T s}}\left|\hat{\gamma}_{k 1}(f)\right|^{2} d f+2 N_{0} \cdot \int_{\frac{1}{T s}} \hat{\gamma}_{11}(f) d f
\end{aligned}
$$

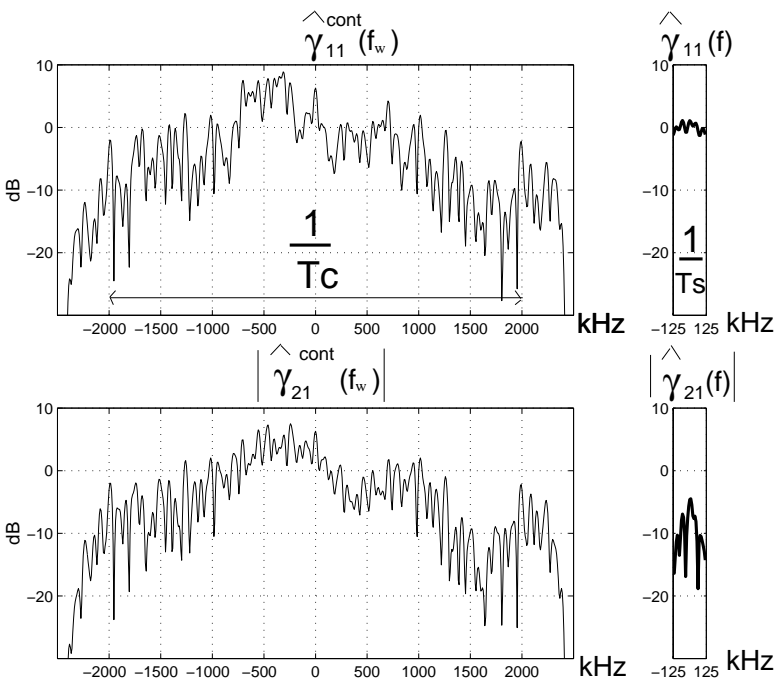

Fig. 3.- $\mathrm{Tx} / \mathrm{Rx}$ Global Channel example in VB. Exemple de canaux globaux $E / R$ en $V B$.

The power of ISI is derived from the quadratic frequency area between the desired aT/RGC and an ideal flat (white) one: it results thus from the nonwhiteness of the desired aT/RGC. The power of MAI is derived from the sum of the quadratic frequency area of interfering aT / RGC relative to the branch "1". The power of Noise is derived from the Noise Equivalent Bandwidth $\gamma_{11[0]} \cdot \frac{1}{T s}$ of the branch "1", i.e. from the frequency area in the symbol band of the desired aT/RGC.

\section{IV.3. Equivalent matrix models}

For compacting and simplifying the representation of the downlink CDMA system, we can use two equivalent matrix models, named wide-band model and symbol-band model. The diversity can well be represented by the wide-band model, by showing the $Q_{r}$ bands containing the received signal. It may be useful when some parameters (channel, codes, timing synchronization) are not identified. Otherwise, being the context of this article, the representation model in the symbol band will be sufficient to establish the expressions and performances of the equalizers.

\section{IV.3.1. Wide-band model}

The $Q_{r}$ components of the received signal at the frequency $f$ in the different bands (equations 13 and 14) are expressed algebraically by:

$$
\underline{\hat{r}}(f)=\underline{\underline{G}}(f) \underline{\hat{a}}(f)+\underline{\hat{n}}(f)
$$

where

$$
\begin{aligned}
& \underline{\underline{G}}(f) \triangleq\left[\underline{\hat{g}}_{1}(f), \ldots, \underline{\hat{g}}_{K}(f)\right], \\
& \underline{\hat{g}}_{k}(f)=\left[\hat{g}_{k}\left(f-\frac{Q_{r / 2}}{T s}\right), \hat{g}_{k}\left({ }^{\prime \prime \prime \prime}+\frac{1}{T s}\right) \ldots, \hat{g}_{k}\left(f+\frac{Q_{r / 2}}{T s}\right)\right]^{T}, \\
& \underline{\hat{r}}(f)=\left[\hat{r}\left(f-\frac{Q_{r / 2}}{T s}\right), \ldots, \hat{r}\left(f+\frac{Q_{r / 2}}{T s}\right)\right]^{T}, \\
& \underline{\hat{n}}(f)=\left[\hat{n}\left(f-\frac{Q_{r / 2}}{T s}\right), \ldots, \hat{n}\left(f+\frac{Q_{r / 2}}{T s}\right)\right]^{T}, \\
& \underline{\hat{a}}(f)=\left[\hat{a}_{1}(f), \ldots, \hat{a}_{K}(f)\right]^{T} .
\end{aligned}
$$


The matrix $\underline{G}(f)$ of size $Q_{r} \times K$ contains the wideband waveforms relative to $f$ in the different symbolbands, and for the different users. It plays the role of a transfer matrix from the $K$ sources towards the $Q_{r}$ bands, for a given frequency of the symbol-band.

An analogy can be made with the source separation from the reception on an array of $Q_{r}$ sensors (in place of $Q_{r}$ frequency bands). In antenna processing domain $[19,8]$, each of the $Q_{r}$ sensors receives a mixture of $K$ sources but the latters arrive from different directional (spatial) vectors. In CDMA, each of the $Q_{r}$ symbol-bands of the reception contains a mixture of the $K$ sources (each band containing all the information for a given source) but the sources "arrive" from different "frequency vectors", imposed by the codes and the channel.

We find again some features which are also classical assumptions in antenna processing:

- the noise is uncorrelated from one band to another: $\frac{1}{T_{s l o t}} \cdot E\left\{\underline{\hat{n}}(f) \underline{\hat{n}}^{H}(f)\right\}=2 N_{0} \cdot \underline{\underline{I}}_{Q_{r}}$,

- the uncorrelation between the sources leads to: $\frac{1}{T_{s l o t}} \cdot E\left\{\underline{\hat{a}}(f) \underline{\hat{a}}^{H}(f)\right\}=\left(A^{2} T s\right) \cdot \underline{\underline{I}}_{K}$,

where $\underline{I}_{N}$ denotes the identity matrix of size $N \times N$.

The beamforming privileges one spatial direction for extracting the source "1" from the coherent recombining of the outputs of the $Q_{r}$ sensors. In the same way in CDMA, the matched filter privileges one "frequency signature" by coherently (in branch "1" for the desired user) re-combining the components of the $Q_{r}$ bands or sub-carriers, which appears in (4) and can be formulated as:

$$
\begin{aligned}
\underline{\hat{y}}(f) & =\underline{\underline{G}}^{H}(f) \underline{\hat{r}}(f) \\
& =\underline{\underline{G}}^{H}(f) \underline{\underline{G}}(f) \underline{\hat{a}}(f)+\underline{\underline{G}}^{H}(f) \underline{\hat{n}}(f)
\end{aligned}
$$

where

$\underline{\hat{y}}(f)=\left[\hat{y}_{1}(f), \ldots, \hat{y}_{K}(f)\right]^{T}$.

The equivalent diagram of transmission and reception for the wide-band model is given in figure 4 :

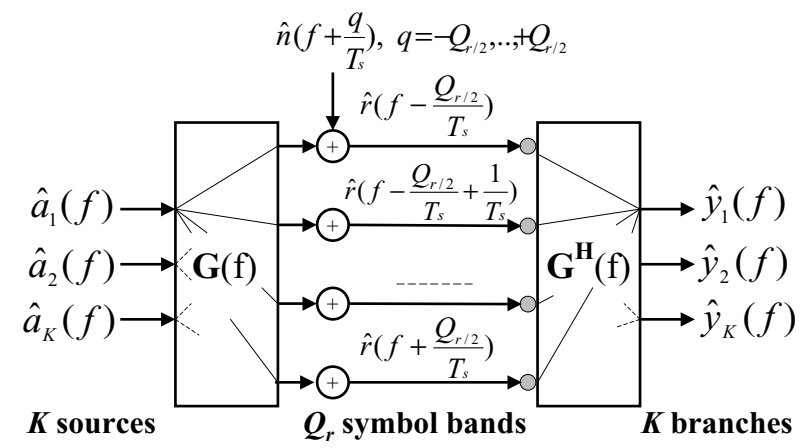

Fig. 4.- Transmission multi-band model. Modèle de la transmission multi-bande.

\section{IV.3.2. Symbol band model}

Spectral matrix of the aliased cross-channels We define, for each frequency $f$ belonging to $\left[-\frac{1}{2 T s}\right.$, $+\frac{1}{2 T s}[$, the normalized spectral matrix of the crosschannels aliased in the symbol band. This matrix groups all the aT/RGC in the following form:

$$
\underline{\underline{\Gamma}}(f) \triangleq \frac{1}{\gamma_{11[0]}} \cdot\left(\begin{array}{cccc}
\hat{\gamma}_{11}(f) & \hat{\gamma}_{21}(f) & \ldots & \hat{\gamma}_{K 1}(f) \\
\hat{\gamma}_{12}(f) & \hat{\gamma}_{22}(f) & \ldots & \hat{\gamma}_{K 2}(f) \\
\hat{\gamma}_{1 K}(f) & \hat{\gamma}_{2 K}(f) & \ldots & \hat{\gamma}_{K K}(f)
\end{array}\right)
$$

This matrix is of a $K \times K$ size, hermitian and nonnegative definite. It will be with full rank and so positive definite for all $f$ if the spectrum $\left|\hat{h}\left(f_{w}\right)\right|^{2}$ of the propagation channel does not have zero in the wideband. Note that the absence of zero is a sufficient but not a necessary condition. On the contrary, if the spectrum of the propagation channel has a common zero at a same frequency $f$ in more than $\left(Q_{r}-K\right)$ different symbol-bands, the matrix is necessarily with rank deficient for this specific frequency. Therefore, excepted for pathological channels, the normal rank (i.e. for almost all $f$ ) will be full, equal to $K$.

In terms of interference for the whole users, ISI corresponds to the "non-whiteness" of the diagonal terms of $\underline{\underline{\Gamma}}(f)$, and MAI to the non zero non diagonal terms.

The formulation of $\underline{\underline{\Gamma}}(f)$ from $\underline{\underline{G}}(f)$ is expressed with a matrix form from the aliasing relation (16):

$$
\underline{\underline{\Gamma}}(f)=\frac{1}{\gamma_{11[0]}} \cdot \underline{\underline{G}}^{H}(f) \underline{\underline{G}}(f)
$$

\section{matrix form of the model}

The symbol band model (figure 5) links directly the $K$ sources of symbols to the outputs of the $K$ branches via the transfer matrix $\underline{\underline{\Gamma}}(f)$. It can be derived from the figure 4 (i.e. equation (22)) of the wide-band model or directly from the equation (5) after passage through frequency and matrix formulation:

$$
\underline{\hat{y}}(f)=\gamma_{11[0]} \cdot \underline{\underline{\Gamma}}(f) \underline{\hat{a}}(f)+\underline{\hat{\eta}}(f)
$$

where

$\underline{\hat{\eta}}(f)=\left[\hat{\eta}_{1}(f), \ldots, \hat{\eta}_{K}(f)\right]^{T}=\underline{\underline{G}}^{H}(f) \underline{\hat{n}}(f)$.

The equivalent discrete noise $\eta_{k[m]}$, artificially reported in addition on each branch ouptut is gaussian, correlated temporally and from one branch to another. Its cross-spectral density, from the branch $" i "$ to the branch " $k$ " is $\frac{E\left\{\hat{\eta}_{i}(f) \cdot \hat{\eta}_{k}^{*}(f)\right\}}{T_{s l o t}}=2 N_{0} \cdot \hat{\gamma}_{i k}(f)$, that is under matrix form:

$$
\frac{1}{T_{s l o t}} \cdot E\left\{\underline{\hat{p}}(f) \underline{\hat{q}}^{H}(f)\right\}=2 N_{0} \cdot \gamma_{11[0]} \cdot \underline{\underline{\Gamma}}(f)
$$

Two examples of the matrix $|\underline{\underline{\Gamma}}(f)|$ are given for $K=8$ users (with linear scales) : in figure 6 , the temporal spreading of the channel (one realization of the Pedestrian B channel according to the ETSI model) is around $1 T s$. 


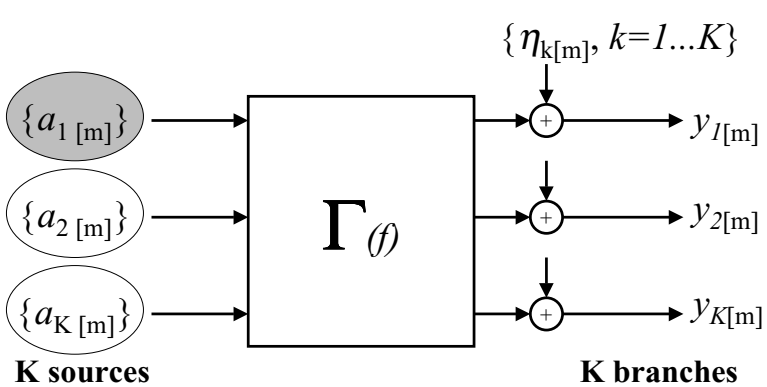

Fig. 5.- Symbol-band model of the transmission. Modèle 'bande symbole' de la transmission.

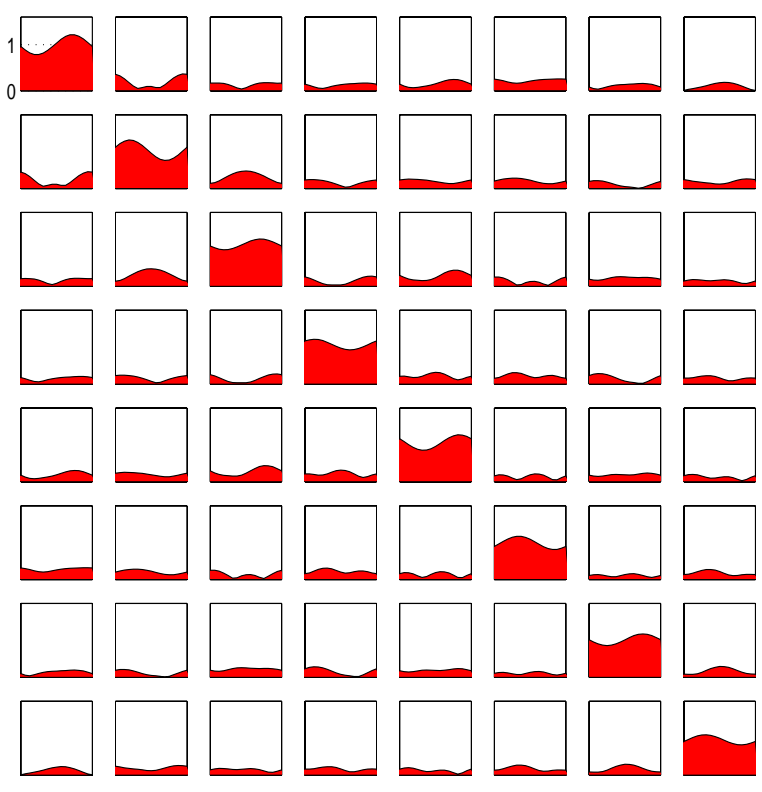

Fig. 6.- Matrix $|\underline{\underline{\Gamma}}(f)|$ example in Pedestrian B. Exemple de $\bar{m}$ atrice $|\underline{\underline{\Gamma}}(f)|$ en Pedestrian $B$.

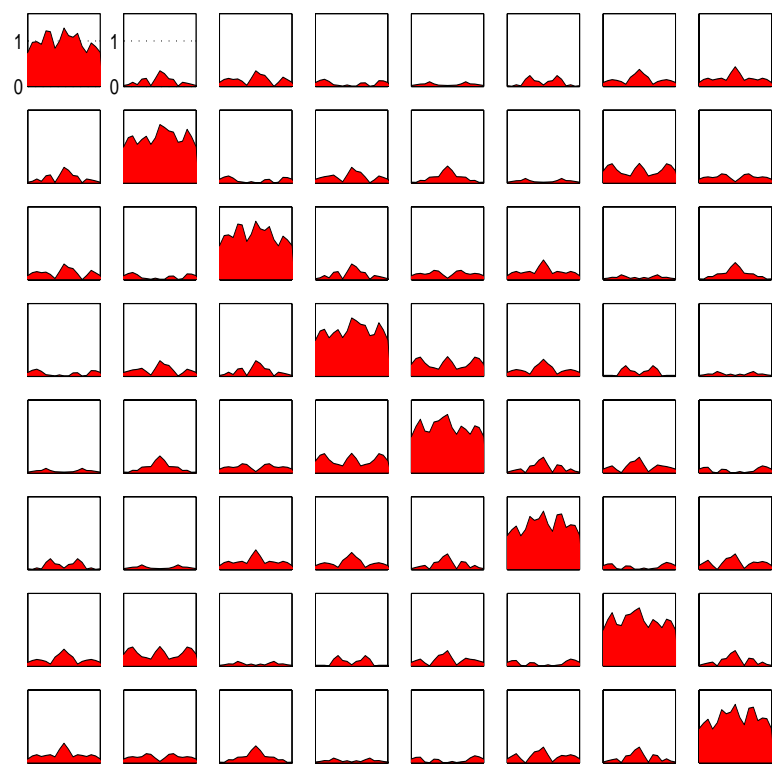

Fig. 7.- Matrix $|\underline{\underline{\Gamma}}(f)|$ example in Vehicular B. Exemple de $\bar{m}$ atrice $|\underline{\underline{\Gamma}}(f)|$ en Vehicular $B$.
In figure 7 , the temporal spreading is around 5 Ts (same Vehicular B channel than used in figure 3 ). In the two examples, the interference is around the same order but we can verify that the coherence bandwidth in Vehicular B is about 5 times smallest.

\section{EXPRESSIONS AND PERFORMANCES OF LINEAR DETECTORS}

\section{V.1. Matched Filter only}

Signal to Interference and Noise Ratio

The matched filter has the feature of maximizing the Signal to Noise Ratio (SNR) at $m T s$ times, without considering the interference (ISI and MAI). To take the interference into account, the quality criterion of the Signal to Interference and Noise Ratio (SINR) is best appropriate.

For the desired user ("1"), the SINR in output of the first branch of the matched filter bank is expressed from the matrix $\underline{\underline{\Gamma}}(f)$ by:

$$
\begin{aligned}
& \begin{aligned}
(S I N R)_{M F} & =\frac{A^{2} \gamma_{11[0]}^{2}}{(I N)} \\
& =\left(\frac{2 E_{b}}{N_{0}}\right) \cdot \frac{1}{1+\left(\frac{2 E_{b}}{N_{0}}\right) \underline{\Sigma}^{T} \operatorname{Ts} \int_{\frac{1}{T s}} \mid \underline{\underline{\Gamma}(f) \underline{\mathbf{1}}-\left.\underline{\mathbf{1}}\right|^{2} d f}}
\end{aligned} \\
& \text { where } \underline{\Sigma}^{T} \triangleq[1, \cdots, 1], \underline{\underline{\mathbf{1}}}^{T} \triangleq[1,0, \cdots, 0]
\end{aligned}
$$

In the absence of ISI and MAI, the matched filter obtains the best SINR equal to $\left(\frac{2 E_{b}}{N_{0}}\right)$ as well as the ideal elementary Binary Error Probability.

\section{V.2. Equalizers: ZF AND MMSE}

In the presence of interference, a numerical linear equalizer bank running at $T s$ completes the head of the base-band receiver, as set forth in paragraph III.2..

The decision variable $d_{1[m]}$ defined in (8) can be written as a global convolutive discrete transfer $\left\{g \operatorname{lo}_{1 i[n]}, i=1 \ldots K\right\}$ for the symbols, disturbed by an additive discrete noise $b_{1}$ :

$$
d_{1[m]}=\sum_{i=1}^{K}\left(g l o_{1 i} * a_{i}\right)_{[m]}+b_{1[m]}
$$

with glo $_{1 i[n]} \triangleq \frac{1}{\gamma_{11[0]}} \sum_{k=1}^{K}\left(e_{1 k} * \gamma_{i k}\right)_{[n]}$

The Mean Squared Error (MSE) is generally used to describe the performances of the equalizer. This energetic measure benefits from a simple quadratic formulation and presents simple solutions of minimization problems. The MSE in the output of the equalizer for the desired user is expressed in frequency terms by: 
$(M S E)_{E q} \triangleq E\left\{\left|d_{1[m]}-a_{1[m]}\right|^{2}\right\} \quad$ to $g l_{1_{k[n]}}=\delta_{k 1} \delta_{n}$ in discrete time representation.

$=\mathrm{A}^{2} \mathrm{Ts} \int_{\frac{1}{T s}}\left|g \hat{l}_{o_{11}}(f)-1\right|^{2} d f$

$+\mathrm{A}^{2} \mathrm{Ts} \sum_{k=2}^{K} \int_{\frac{1}{T s}}\left|g \hat{l}_{o_{1 k}}(f)\right|^{2} d f+2 N_{0} \cdot \int_{\frac{1}{T s}}\left|\hat{t}_{1}(f)\right|^{2} d f$

The integration term in this expression is the power spectral density of the error (psd), comprised of a ISI term, a MAI term, and a noise term.

From matrix formulation:

- the vector $\underline{\hat{e}}_{1}^{T}(f)=\left[\hat{e}_{11}(f), \cdots, \hat{e}_{1 K}(f)\right]$ is made from the transfer functions of the equalizing filters on the $K$ branches,

- the global transfer function for the symbols of the user $k$ is: $\quad g \hat{l}_{o_{1 k}}(f)=\underline{\hat{e}}_{1}^{T}(f) \cdot \underline{\underline{\Gamma}}(f) \cdot \underline{\mathbf{k}}$ where $\underline{\mathbf{k}}^{T}=[\underbrace{\cdots, 0,1}_{k}, 0, \cdots]$

- the global transfer function $\hat{t}_{1}(f)$ for the noise (with periodicity $\frac{1}{T s}$ due to the sampling) is such that: $\left|\hat{t}_{1}(f)\right|^{2}=\frac{1}{\gamma_{11[0]}} \underline{\hat{e}}_{1}^{H}(f) \cdot \underline{\underline{\Gamma}}^{*}(f) \cdot \underline{\hat{e}_{1}}(f)$.

The psd of $b_{1[m]}$ is then $2 N_{0} \cdot\left|\hat{t}_{1}(f)\right|^{2}$.

From these expressions, it is easy to establish, by omitting $(f)$ in the second member for the sake of clarity, that:

$$
\frac{p s d}{\mathrm{~A}^{2} \mathrm{Ts}}(f)=\underline{\hat{e}}^{H}\left(\underline{\underline{\Gamma}}^{*} \underline{\underline{\Gamma}}^{*}+\frac{N_{0}}{2 E_{b}} \cdot \underline{\underline{\Gamma}}^{*}\right) \underline{\hat{e}_{1}}-2 \operatorname{Re}\left\{\underline{\hat{e}}^{H} \underline{\underline{\Gamma}}^{*} \underline{\mathbf{1}}\right\}+1
$$

Notes:

* the power of interference plus noise can be obtained simply from the MSE by subtracting the quadratic error term at zero delay: $(I N)_{E q}=(M S E)_{E q}-A^{2}\left|1-g l o_{11[0]}\right|^{2}$

* The global linear detector $l_{1}$ for the detection of the symbols of user "1", including the MF-sampler bank and the equalizing filter bank, has a transfer function $\hat{l}_{1}\left(f_{w}\right)$ defined for the wide-band. It achieves actually, for one frequency $f$ of the symbol-band, a linear combination of the $Q_{r}$ sub-bands. Its expression under vector form of size $Q_{r}$ (grouping $\hat{l}_{1}\left(f-\frac{Q_{r / 2}}{T s}\right), \ldots \hat{l}_{1}\left(f+\frac{Q_{r / 2}}{T s}\right)$, is:

$$
\underline{\hat{l}}^{T}(f)=\frac{1}{\gamma_{11[0]}} \cdot \underline{\hat{e}}_{1}^{T}(f) \underline{\underline{G}}^{H}(f)
$$

\section{V.2.1. Zero Forcing equalizer}

The optimal Zero-Forcing equalizer minimizes the MSE, under the constraint of cancelling the ISI and MAI. With such a criterion, in situations without noise, the estimated symbols must be equal to the transmitted symbols for the desired user. In the same time, among all the wide-band global filters $l_{1}$ which cancel the interference, this criterion warrants the one with a minimal norm and leads to the structure on figure 2 (or equation (27)). The cancelling of the interference from the symbol band model requires then that $g \hat{l}_{1 k}(f)=\delta_{k 1}$, which is equivalent
The equalizer vector is then obtained by inverting the spectral matrix of aliased cross-channels:

$$
\underline{\hat{e}}_{1}^{T} z f(f)=\underline{\mathbf{1}}^{T}[\underline{\underline{\Gamma}}(f)]^{-1}
$$

This is immediately derived from the equation (23) of the symbol-band model. So, the resulting MSE is formed only by the contribution of the noise, amplified by the interference cancelling process:

$$
(M S E)_{z f}=\left(2 N_{0} \cdot \frac{1}{T s}\right) \cdot \frac{1}{\gamma_{11[0]}} \cdot e_{11 z f[0]}
$$

The zero delay coefficient of the equalizer in branch "1", $e_{11 z f[0]}$, is a positive real number, derived from diagonals of Hermitian matrix. It plays a fundamental role since it describes entirely the performance of the system. More precisely, it expresses a degradation factor for the SINR but also for the Error Probability (Pe or BER ${ }^{2}$ since after perfect theoretical equalization, we obtain exactly the desired symbols, just disturbed by a gaussian noise:

$$
\begin{gathered}
(S I N R)_{z f}=\frac{A^{2}}{(M S E)_{z f}}=\left(\frac{2 E_{b}}{N_{0}}\right) \cdot \frac{1}{e_{11 z f[0]}} \\
(P e)_{z f}=Q\left(\sqrt{\left(\frac{2 E_{b}}{N_{0}}\right) \cdot \frac{1}{e_{11 z f}[0]}}\right)
\end{gathered}
$$

The degradation factor corresponds to the frequencydomain area of the first diagonal "block" (or frequency pattern) of the inverted aliased cross-spectral matrix:

$$
e_{11 z f[0]}=T s \int_{\frac{1}{T s}}\left[\underline{\Gamma}(f)^{-1}\right]_{11} d f
$$

The Schwartz inequality lets us verify that $e_{11 z f[0]} \geq 1$. Of course, no degradation $\left(e_{11 z f[0]}=1\right)$ corresponds to an ideal situation with a single path channel and orthogonal codes.

Figure 8 gives the example of an inverted matrix $\left|\underline{\underline{\Gamma}}(f)^{-1}\right|$ in Pedestrian B. The patterns of the first line represent then the frequency modulus of the equalizing filters $\hat{e}_{1 k}(f)$ for $k=1$ to 8 ; the degradation factor for the desired user " 1 " is rather weak, computed to $+1.2 \mathrm{~dB}$.

Note: From the received signal $r(t)$, there are an infinity of linear detectors $l_{1}$ able to cancel interference. The linear ZF equalizer recalled here (with theoretical frequency description and therefore no constraint of finite

\footnotetext{
${ }^{2}$ the function $Q($.$) measures the area under the tail of a$ Gaussian distribution: $Q(x)=\frac{1}{\sqrt{2 \pi}} \int_{x}^{\infty} e^{-u^{2} / 2} d u$.
} 


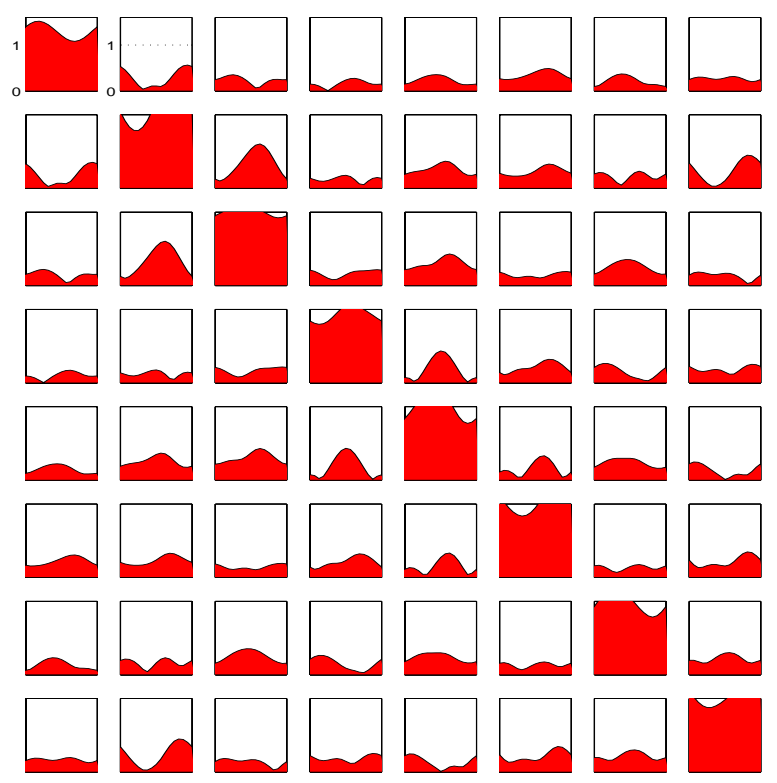

Fig. 8.- Matrix $\left|\underline{\underline{\Gamma}}(f)^{-1}\right|$ example in Pedestrian B. Exemple de matrice $\left|\underline{\underline{\Gamma}}(f)^{-1}\right|$ en $P B$.

length for the impulse responses) is the optimal since it cancels interference but also minimizes, under this constraint, the noise amplification. It is not the case for a $\mathrm{ZF}$ detector which would consist in inverting the wide-band continuous channel, in the bandwidth of the RRC, before making the correlation with the desired code. Such a detector would generally present much worse performances.

\section{V.2.2. MMSE equalizer}

The mean squared error is minimum when the quadratic form (26) of the spectral density of the error is minimized for each frequency $f$ of the symbol band, which leads to:

$$
\underline{\hat{e}}_{m e}^{T}(f)=\underline{\mathbf{1}}^{T}\left[\underline{\underline{\Gamma}}^{\prime}(f)\right]^{-1}
$$

with $\underline{\underline{\Gamma}}(f)=\underline{\underline{\Gamma}}(f)+\left(\frac{N_{0}}{2 E_{b}}\right) \cdot \underline{\underline{I}}_{K}$

The expression of the MMSE is then:

$$
\begin{aligned}
(M S E)_{m e} & =A^{2}\left(1-g l o_{11 m e}[0]\right. \\
& =\left(2 N_{0} \frac{1}{T s}\right) \cdot \frac{1}{\gamma_{11[0]}} \cdot e_{11 m e[0]}
\end{aligned}
$$

The inversion of the aliased cross-channels spectral matrix is here realized after adding a noise to signal $\frac{N_{0}}{2 E_{b}}$ factor on the diagonal. So, as in a single-user situation, the partial inversion of the MMSE limits the amplification of the noise at the frequencies where the local signal to noise ratio is bad. Moreover, since the power of the noise is not zero, the inverse of $\underline{\underline{\Gamma}}^{\prime}(f)$ always exists and ill conditioned situation is impossible, contrary to the ZF case.

The global gain (at zero delay) of all the chain $\mathrm{Tx} / \mathrm{Rx}$ for user "1", glo ${ }_{11 m e[0]}=T s \int_{\frac{1}{T s}}\left[\underline{\underline{\Gamma}}^{\prime}(f)^{-1} \underline{\underline{\Gamma}}(f)\right]_{11} d f$, is a positive real less or equal to 1 (equal to 1 in noisefree situation). The MMSE receiver has the same behavior than the $\mathrm{ZF}$ receiver in situations of strong $\frac{E_{b}}{N_{0}}$ and is reduced to the head of receiving, i.e. to the MF, in the extreme opposite situation. The zero delay coefficient of the equalizer is here normalized by the global gain to form the degradation factor of the $\operatorname{SINR}(\geq 1)$, which is expressed through:

$$
\begin{aligned}
(S I N R)_{m e} & =\frac{A^{2} g l o_{11 m_{e}[0]}^{2}}{(M S E)_{m e} g l_{11 m e}[0]} \\
& =\left(\frac{2 E_{b}}{N_{0}}\right) \cdot g l o_{11 m e[0]} \cdot \frac{1}{e_{11 m e[0]}}
\end{aligned}
$$

For strong $\frac{E_{b}}{N_{0}}$ situations, the SINR of the three receivers recalled in this article are ordered in the following manner:

$$
\begin{aligned}
& (S I N R)_{m f} \leq(S I N R)_{z f} \leq(S I N R)_{m e} \leq\left(\frac{2 E_{b}}{N_{0}}\right) \\
& \text { when } \frac{2 E_{b}}{N_{0}}>>1
\end{aligned}
$$

Then, the performances of the receiver are expected to be improved by equalization, MMSE preferentially if the noise level is not negligible.

\section{V.2.3. Comparisons and design elements}

The multi-user equalizer we have just described generalizes the classical results obtained with weakly selective channels, based on the correlation matrix of the codes at zero delay. When the temporal spread of the channel is weak compared to the symbol duration, the equalizer needs no memory dependence (white response in frequency) and is reduced to instantaneously and linearly combining the $y_{k[m]}$. In this case, the spectral matrix of aliased cross-channels, white in frequency, but non diagonal if the coded waveforms are not orthogonal, is identical to the zero delay correlation matrix, as the frequency dimension does not appear. We then find the equalizer described by the authors of $[16,5,6]$, where the combining coefficients $e_{1 k[0]}$ from the branches $k=1$ to $K$ are given by the first line of the inverse of the zero-delay correlation matrix of the codes, equal to $[1,0, . ., 0]$ if the codes are orthogonal.

In the presence of selective aT/RGC in the symbol band, the equalizing filters may use practically a finite temporal depth of $P$ coefficients (with causal and anti-causal part). We then have added the frequency dimension $f$, which is equivalent to independently treating several non selective channels, with narrow bands compared to $\frac{1}{T s}$ (second order stationarity).

This approach naturally (but approximately) provides the coefficients of the equalizer by using the Inverse Discrete Fourier Transform, and constitutes an alternative solution to temporal methods $[12,4]$, with a little complexity. 
To obtain a temporal depth of $P$ coefficients for the equalizer, we just have to sample the symbol band with a step $\Delta f=\frac{1}{P T s}$ to form the $P$ matrix $\underline{\Gamma}(p . \Delta f)$ and compute their inverse. The $K$ noncausal filters $\left\{e_{1 k[p]}\right\}$ used to detect the symbols of user "1" are obtained by a $D F T^{-1}$ of the $K$ spectral patterns of the line "1" of the inverse matrix. The filter $\left\{e_{11[p]}\right\}$ born from the diagonal is the most energetic and corresponds to the main branch. In a multi-code case, i.e. with several desired codes, we can take advantage of the hermitian symmetry of the taps: $e_{i k[n]}=e_{k i[-n]}^{*}, \quad \forall i, k=1 \ldots K, \quad \forall n \in \mathbb{Z}$.

Notes:

* The impulse responses of the equalizer bank at symbol time after MF bank are theoretically infinite. So, the choice of $P$ should ensure a negligible temporal aliasing for the practical filters. According to the weak values of the secondary far paths in the ETSI models described in VII., a typical temporal depth of 1 to 4 coefficients in indoor A and of 8 to 16 coefficients in Vehicular B is generally sufficient.

* Instead of approximately preserving the theoretical imposed structure described in figure 2 (with a finite number of taps for the practical equalizer), it is also possible to compact the linear detector $l_{1}$ in a single discrete transverse fractional filter with a cadence of $T c / 2$ at input and $T s$ at output.

\section{EXTENSION TO MULTI-SENSOR RECEPTION}

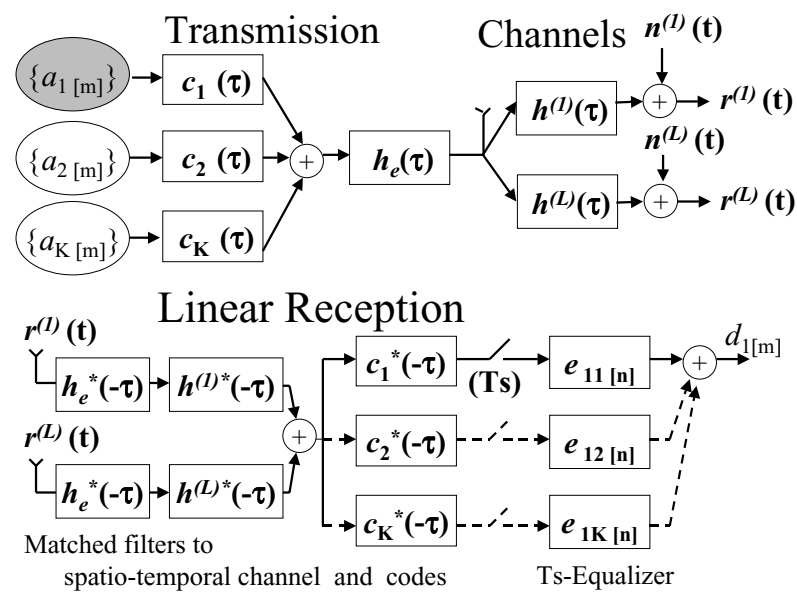

Fig. 9.- Multi-sensor transmission model. Modèle de transmission multi-capteur.

When the mobile receiver uses $L=2$ or 3 sensors (with a spacing above $\lambda / 2=7.5 \mathrm{~cm}$ ), the $i=1 \ldots L_{t}$ propagation paths are observed on the different elements $l=1 \ldots L$ of the antenna. The delays of one path " $i$ " on the different sensors " $l$ " are nearly identical according to chip time (Cf note in section II.), only the complex amplitudes $\alpha_{i}^{l}$ are different. Thus, we define $L$ propagation channels $h^{(l)}(\tau)$ from the model of (3), still considered here as deterministic and time invariant during the slot.

To the $l$-th sensor are also associated a received signal $r^{(l)}(t)$, a spatio-temporally white noise $n^{(l)}(t)$, a wide-band waveform for the $k$-th user $g_{k}{ }^{(l)}(\tau)=\left(c_{k} * h_{e} * h^{(l)}\right)(\tau)$ and a transfer matrix sources/bands $\underline{\underline{G}}^{(l)}(f)$. With multi-sensor, $\gamma_{11[0]}$ and $E_{b}$ represent respectively the normalized "energy" of the waveform and the average energy per bit of the desired signal over the $L$ receiving sensors.

The channel matched filter becomes spatio-temporal $[1,7,11,15]$ but same as the multi-band dimension, the spatial dimension does not appear explicitly in the "symbol-band" model and the digital equalizer keeps exactly the same expressions as function of $\underline{\Gamma}(f)$ than those established in single-sensor, described by equations $(28,29,30,31,32)$. Obviously, the global diversity is concealed in $\underline{\underline{\Gamma}}(f)$ since $|\hat{h}|^{2}$ is replaced by $\sum_{l}\left|\hat{h}^{(l)}\right|^{2}$ in the expression (17) of the $\mathrm{T} / \mathrm{RGC} \hat{\gamma}_{i k}^{\text {cont }}(f)$ and thus indirectly in the formation of the $\operatorname{aT} / \mathrm{RGC} \hat{\gamma}_{i k}(f)$, leading to:

$$
\underline{\underline{\Gamma}}(f)=\frac{1}{\gamma_{11[0]}} \cdot \sum_{l} \underline{\underline{G}}^{(l)}{ }^{H}(f) \underline{\underline{G}}^{(l)}(f)
$$

The coherent re-combination of the spatial and frequency channels improves the orthogonality of the waveforms and therefore makes easier the system inversion operated by the joint detection equalizer. In term of implementation, we can yet approximate (with finite length) the imposed structure of figure 9 , taking benefit of the discrete nature of the paths in the channel MF. Or we can implement linear receivers by means of one single fractional filtering per sensor. We are now going to measure the benefit of the multi-sensor reception in the next section.

\section{AVERAGE PERFORMANCES IN INDOOR AND VEHICULAR}

\section{VII.1. Context}

used codes:

According to the general hypothesis, the codes $\left\{c_{k[q]}, q=0 \ldots Q-1\right\}$ for the users $k=1 \ldots K$ are complex with values proportional to $\{1, j,-1,-j\}$, algebraically independent and moreover, in conformity with the norm [21], algebraically orthogonal (Cf (2)). One code is comprised of $Q=16$ chips obtained from a "channel code" $\left\{c h_{k[q]}, q=0 \ldots Q-1\right\}$ and a "scrambling code" $\left\{v_{[q]}, q=0 \ldots Q-1\right\}$. The channel code is particular to one user, with real binary values $(\in\{-1 ;+1\})$ chosen in the family of 16 orthogonal Walsh-Hadamard sequences. The scrambling code is specific to each cell, with real binary values indexed in the norm. The complex values of the final code are obtained by a rotation of $\pi / 2$ from a chip to another after multiplication of the two previous codes:

$$
c_{k[q]}=c h_{k[q]} \cdot v_{[q]} \cdot(j)^{q+1}, q=0 \ldots Q-1
$$


where $j$ is the pure imaginary number such as $(j)^{2}=-1$.

So, with such a complex code, both real and imaginary parts of the transmitted symbols benefit of the diversity of the In phase and Quadrature carrier modulation. Moreover, during the transmission of one symbol, the constellation at the chip time presents no zero crossing, ensuring small envelope variations for the transmitted modulated signal, of one given user. The computation of the performances are relative to the desired code " 1 " defined by: $c h_{1[q]}=1 \forall q ; \quad \underline{v}=[-1,+1,-1,-1,-1,+1,-1,-1$, $+1,-1,+1,+1,-1,+1,-1,-1]^{T}$

This code " 1 " is then reduced to the complex version of the scrambling code.

\section{propagation channels:}

Models described by ETSI [20] in environments "Indoor A" (IA) and "Vehicular B" (VB) are used. The channel impulse responses are constituted by 6 paths with relative delays (between two adjacent paths) and average power, summarized in the following table:

\begin{tabular}{|c|l|l|l|l|l|l|}
\hline IA (ns) & 0 & 50 & 110 & 170 & 290 & 310 \\
\cline { 2 - 7 }$(\mathrm{dB})$ & 0 & -3 & -10 & -18 & -26 & -32 \\
\hline VB $(\mu \mathrm{s})$ & 0 & 0.3 & 8.9 & 12.9 & 17.1 & 20.0 \\
\cline { 2 - 7 }$(\mathrm{dB})$ & -2.5 & 0 & -13 & -10 & -25 & -16 \\
\hline
\end{tabular}

The temporal spreading is very weak in IA, slightly superior to one chip time, $T c$; on the contrary, the temporal spread is important in $\mathrm{VB}$, around $5 \mathrm{Ts}$, which will permit to give equalizer performances in two very different situations. The propagation delays are quasi-fixed (variation of $\pm 3 \%$ ). The complex amplitudes $\alpha_{i}^{l}$ of the paths are random, distributed with a Rayleigh law for the modulus and uniform law between 0 and $2 \pi$ for the phases. We suppose the complex amplitudes uncorrelated between two paths (index " $i "$ ) and from one sensor to another (index "l"). This last hypothesis, more often admitted [10], is certainly a bit optimistic for the inter-sensor spacing looking at the mobile; we will come back to this point in the performance analysis.

\section{average performances (random channels):}

We are going to evaluate, for one given model of channel, the performances of the equalizers from theoretical formulas $(24,29,30,32)$ developed in section V.. The frequency integrals are approximated by the rectangle method, with a frequency step $\Delta f=\frac{1}{32 T s}$ and a temporal oversampling of $T c / 8$ to form the delays of the paths. These performances take the form $(S I N R)=\left(\frac{2 E_{b}}{N_{0}}\right) \cdot \frac{1}{\beta_{I}}$ where $\beta_{I}$ represents the degradation on the SINR caused by interference for the Matched Filter, Zero-Forcing and MMSE receiver. For the Zero-Forcing equalizer, $\beta_{I}$ also represents the degradation of the BER.

We remind that these performances formulae sup- pose perfect knowledge of the channel and perfect timing synchronization, they give then upper bounds which will inevitably be degraded in real situations. Moreover, they are only valid for a deterministic and time invariant impulse response, which does not correspond to IA and VB models since they are random, mainly from the complex amplitudes $\alpha_{i}^{l}$. Rather than calculating the performances for one specific observed sample of the paths amplitudes (supposed then deterministic, equal for example to their quadratic average values), we are going to give $a v$ erage performances on the whole possible occurence of impulse response. This classical process $[17,10]$ may be used to represent a practical situation with slowly time variant channel: one occurence of $\alpha_{i}^{l}$ is associated to the channel impulse response at a given instant. The channel variation is slow enough to be considered fixed during the occurence and perfectly estimated. So, the interference term $\beta_{I}$, same as the energetic gain of the global waveform $\gamma_{11[0]}$ become random variables. We are now interested in the expected values of the SINR inverse (named INSR) and of the Error Probability (for ZF), computed from a very large number of sampling:

$$
\begin{gathered}
\overline{(I N S R)} \triangleq E\left(\frac{1}{S I N R}\right)=\left(\frac{N_{0}}{2 \bar{E}_{b}}\right) \cdot\left(\overline{\beta_{I} \cdot \beta_{f}}\right) \\
(\bar{P} e)_{z f}=E\left\{Q\left(\sqrt{\left(\frac{2 \bar{E}_{b}}{N_{0}}\right) \cdot \frac{1}{\beta_{I}} \cdot \frac{1}{\beta_{f}}}\right)\right\}
\end{gathered}
$$

where $\beta_{f} \triangleq \frac{\bar{\gamma}_{11[0]}}{\gamma_{11[0]}}=\frac{\bar{E}_{b}}{E_{b}}$ expresses the amplitude fading, the on-lining representing the expected or average value. The degradation is due to two different phenomena: the interference, through $\beta_{I}$, and the amplitude fading (spreading of the range of the probability density function of the normalized energy $\left.\frac{E_{b}}{\bar{E}_{b}}\right)$, through $\beta_{f}$.

In order to obtain the average degradation only caused by the interference, a situation without fading is artificially introduced. In this situation, from one occurrence to another, the waveform is still random, but its energetic gain $\gamma_{11[0]}$ is maintained at a constant value $\bar{\gamma}_{11[0]}$, which fixes the receive average bit energy to $E_{b}=\bar{E}_{b}$ and makes deterministic $\beta_{f}=\bar{\beta}_{f}=1$.

In situation with fading, only the BER performances in Zero-Forcing are plotted since the $\overline{I N S R}$ curves are practically derived from those of the situation without fading, by an additional weighting factor $\bar{\beta}_{f}$, superior or equal to 1 . This last observation is explained by equation (33) and by the weak correlation between the fading term $\beta_{f}$ and the interference term $\beta_{I}$, as shown in figure 10 which plots the correlation coefficient related to the number of 
users, from the model vB. $\bar{\beta}_{f}$ is thus an indicator of the degradation due to the fading, even if it is well known that the average energetic indexes are not sufficient to express quantitatively the Error Probability. Without interference $\left(\beta_{I}=1\right)$, with a singlepath channel subject to a Rayleigh fading, and with uncorrelated envelopes from one sensor to another, Pe asymptotically varies [17] as $\left(\frac{\bar{E}_{b}}{N_{0}}\right)^{-L}$. The slope of the BER curve (with classical Log scales) is imposed by the diversity order, equal to the number of sensors in this basic example.

With any random channel, we may still define an equivalent diversity order $N_{e q}$ relative to a Rayleigh fading, as proposed in [9], from the variation coefficient $c v$ of the received power:

$$
N_{e q}=\frac{1}{c v^{2}} \quad \text { with } c v=\frac{\sqrt{\overline{E_{b}^{2}}-\bar{E}_{b}^{2}}}{\bar{E}_{b}}
$$

We then dispose of two indexes to measure the depth of the fading: $c v$ and $\bar{\beta}_{f}$. Relatively to a Rayleigh fading with $N$ independent diversity branches (with same power), it can be shown that:

$$
\bar{\beta}_{f}=\frac{N^{N}}{N-1}\left(\frac{1}{N}\right)^{(N-1)}
$$

In order to facilitate the interpretation of the results, we indicate in the following table the values (in decibel) of these two indexes for some diversity orders:

\begin{tabular}{|l||l|l|l|l|l|l|}
\hline$N_{e q}$ & 1 & 2 & 3 & 5 & 8 & $+\infty$ \\
\hline$c v(\mathrm{~dB})$ & 0 & -1.5 & -2.3 & -3.5 & -4.5 & $-\infty$ \\
\hline $\bar{\beta}_{f}(\mathrm{~dB})$ & $+\infty$ & 3 & 1.76 & 0.96 & 0.57 & 0 \\
\hline
\end{tabular}

\section{VII.2. Performance analysis}

\section{VII.2.1. Vehicular without fading}

Figure 11 presents the results in VB with 1, 2, 3 sensors for a situation without fading. Each elementary cell of the figure shows the results superimposed for $1,2,4,8,12$ and 16 users in continuous line, from bottom to top. The dotted lines give the theoretical ideal performances obtained with a single path channel without fading.

Concerning the $(\overline{I N S R})$, we first observe a great improvement (diminution of $\overline{\beta_{I}}$ ) in MMSE or ZF (equivalent to MMSE for strong $\left.\frac{E_{b}}{N_{0}}\right)$ as compared to the single matched filter. For the MF, the $(\overline{I N S R})$ curves reach a floor, for strong $\frac{E_{b}}{N_{0}}$ and for the large values of $K$, because of the interference. The improvement due to the equalization is emphasized with 2 or 3 sensors, which brings almost back to ideal performances.

Concerning the BER in $\mathrm{ZF}$, there is an important loss, around $15 \mathrm{~dB}$ at $\mathrm{Pe}=10^{-3}$ when the saturation of the number of users $(K=16)$ is approached. The multisensor reception gives new degree of freedom and reduces the degradation to just $1 \mathrm{~dB}$ with 3 sensors.

\section{VII.2.2. Vehicular with fading}

Figure 12 presents the results for a $\mathrm{ZF}$ receiver in a fading situation (top of the figure). The upper dotted lines correspond to the performance with a single-path channel subject to Rayleigh fading with only one receiving sensor, i.e. without diversity. The importance of the fading process is embodied by the bottom plots which indicate the scattering of the probability density of the channel energy, related to its average value. We also have mentioned the indexes $c v$ and $\bar{\beta}_{f}$.

We observe with a single-sensor, a degradation of 3 to $5 \mathrm{~dB}$ compared to the curves of BER in without fading situation. The very important degradation above $18 \mathrm{~dB}$ for 16 users is reduced to $3 \mathrm{~dB}$ with 3 sensors.

We notice, for one user and no spatial diversity, that the BER sensitivity is better than in a single path fading situation. This is naturally explained by the path diversity (resolved paths) of the Vehicular environment. Indeed we have for a single-sensor $c v=-1.6 \mathrm{~dB}$ and $\bar{\beta}_{f}=2 \mathrm{~dB}$, which already corresponds to a diversity order $N_{e q}$ between 2 and 3 . This order becomes around 5 and 7 with 2 and 3 sensors.

\section{VII.2.3. Indoor with fading}

It is interesting to compare the fading situation previously noticed to those observed in Indoor A environment (figure 13) where there is almost no path diversity and very few interferences in the MF outputs. With a single sensor, the error probability is nearly equivalent to the one path fading situation for any number of users, expressing weak $\beta_{I}$ and strong $\bar{\beta}_{f}$. The spatial diversity is then required, even if $\mathrm{K}$ is little, in order to decrease the range of the amplitude fading: for one single sensor, $c v=-0.3 \mathrm{~dB}$ and $\bar{\beta}_{f}=5$ $\mathrm{dB}$, which corresponds to a diversity order $N_{e q}$ very little, slightly above 1 . This order becomes around 2.5 and 4 respectively with 2 and 3 sensors.

This improvement would be less significant if the paths were correlated from one sensor to another. Nevertheless, the Indoor environment, which mostly needs spatial diversity, presents a very favourable situation in downlink reception on the mobile [18, 9], due to the proximity between the scattering objects and the receiver. In this case, one path can be modeled by a lot of micro-paths arriving on the mobile sensor with quasily the same delay. According to the model developed in [18] for one macro-path, the correlation between any field component envelope measured at two separate points on the mobile is shown to be weak with a spatial separation above $\frac{\lambda}{2}$. On the contrary, the reception situation on the base station in "uplink" would be very different because of a geometrical asymmetry and would require a spacing above $50 \lambda$ for obtaining weak correlations. 

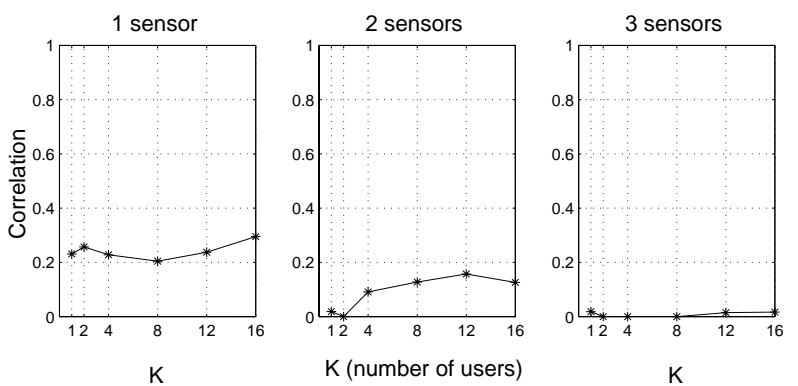

Fig. 10.- Correlation coefficient between $\beta_{I}$ et $\beta_{f}$, computed with the Vehicular B channel model. Coef. de corrélation entre $\beta_{I}$ et $\beta_{f}$ en $V B$.
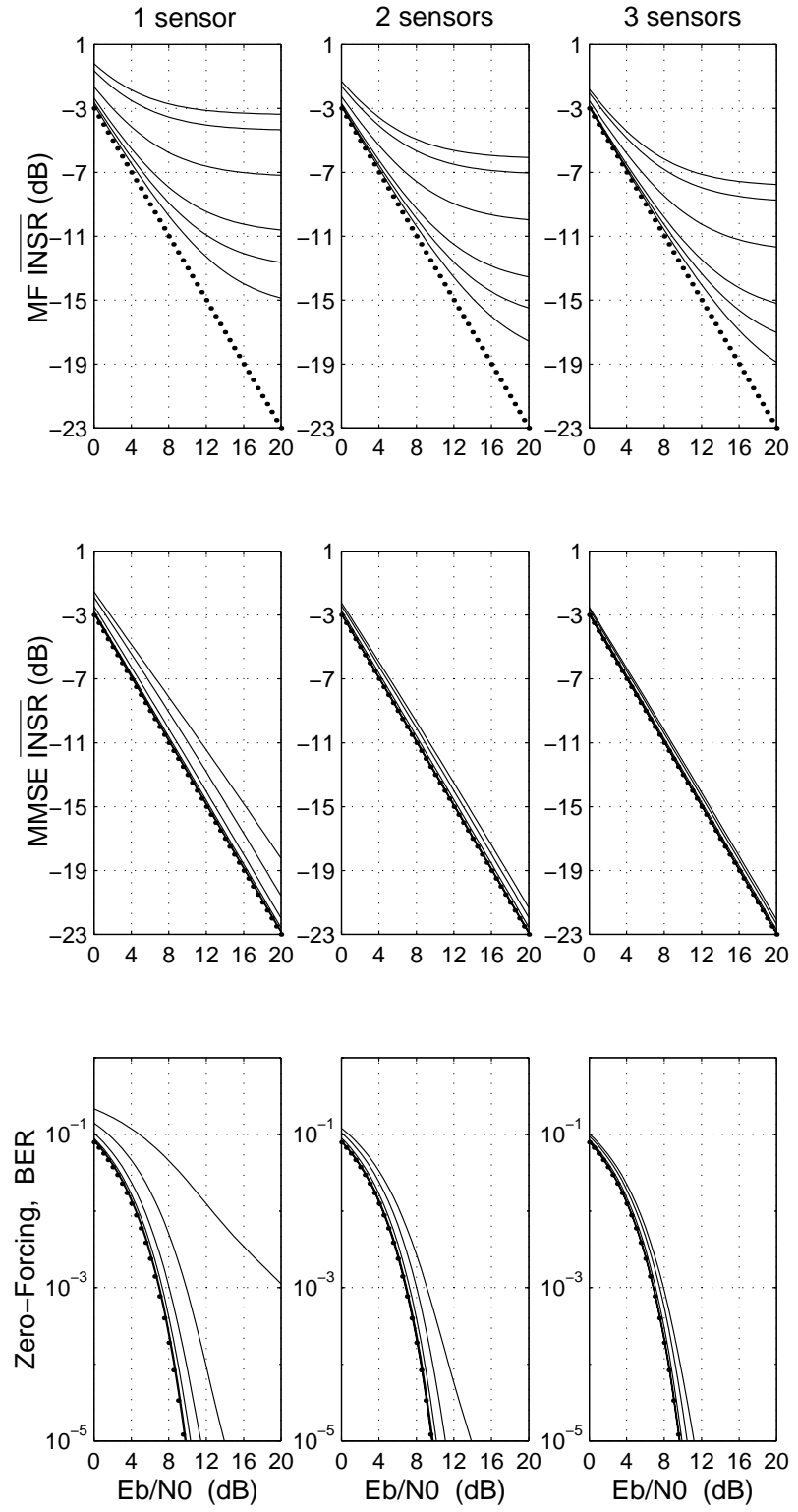

Fig. 11.- Vehicular B performances without fading. Performances en Vehicular B sans fading.
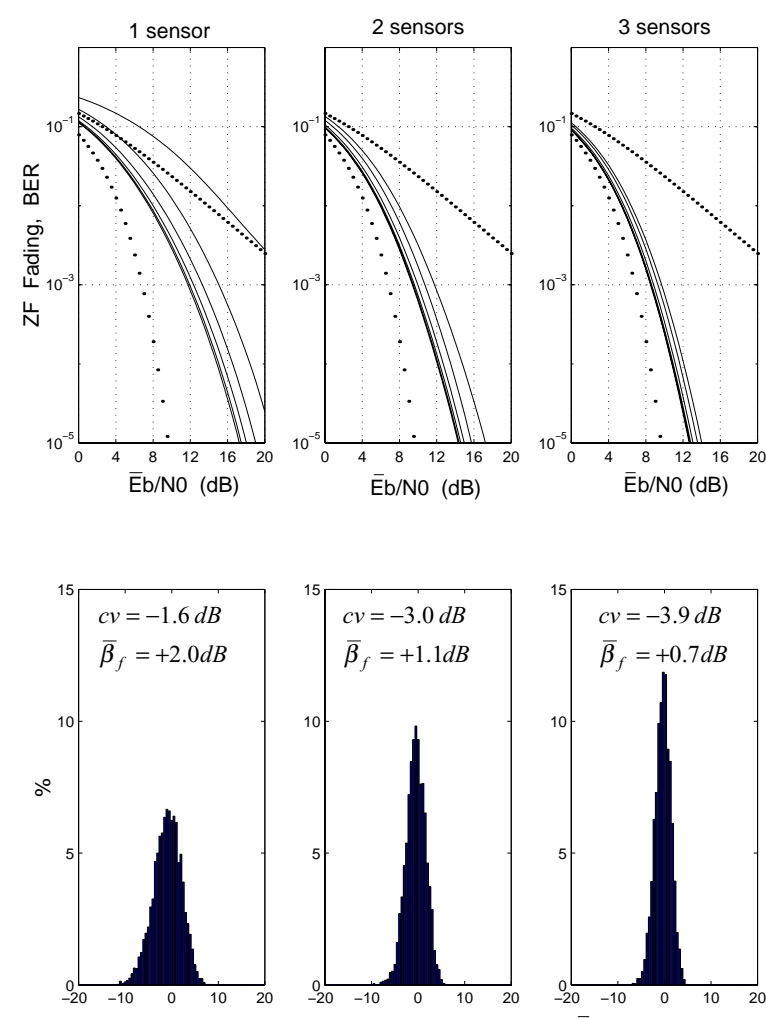

range of normalized received power $\mathrm{Eb} / \overline{\mathrm{E}} \mathrm{b}(\mathrm{dB})$

Fig. 12.- Vehicular B performances with fading. Performances en Vehicular B avec fading.
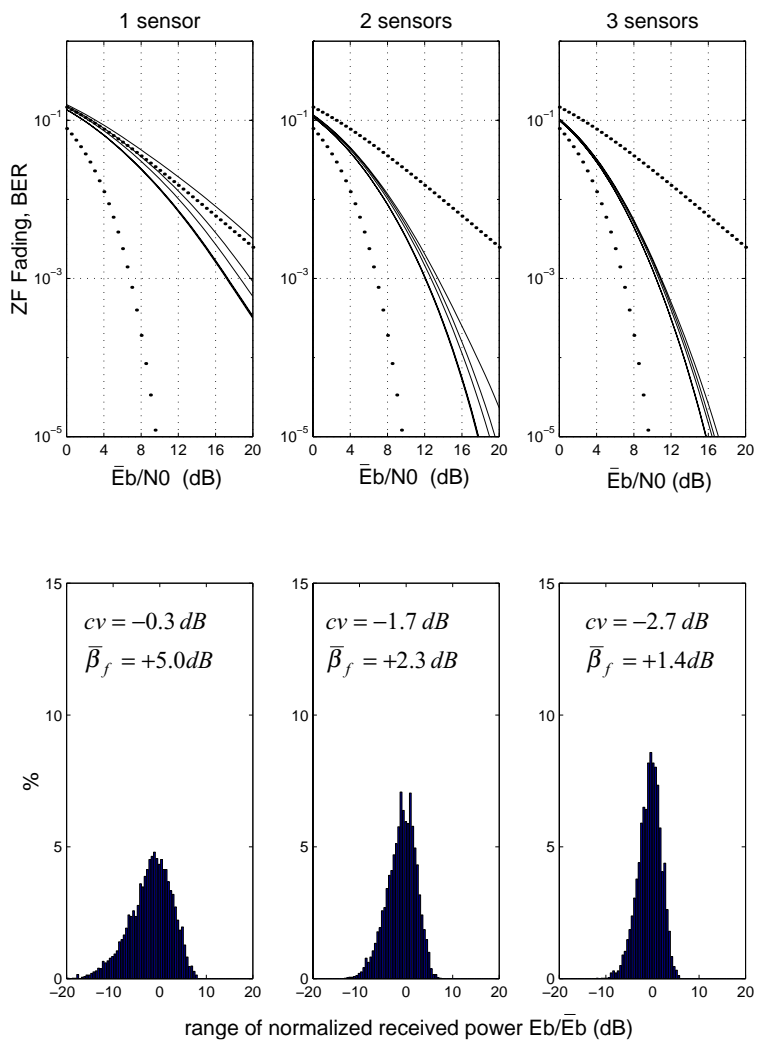

Fig. 13.- Indoor A performances with fading. Performances en Indoor A avec fading. 
In conclusion, a coherent multi-element antenna in reception improves the transmission by fighting against both frequency selectivity and amplitude fading.

Note: in terms of link budget, the presented performances should benefit from an additional improvement of $10 \log (L) \mathrm{dB}$ due to the antenna gain. All the curves were plotted in function of the global energy of all the sensors.

\section{CONCLUSION}

This study has permitted, by taking an approach of frequency interpretations, to recall non exhaustively the linear detection structures in the downlink of a TD-CDMA system. We have formulated the expressions and performances of this detector from the representation in the symbol-band, based on the spectral matrix of the aliased cross-channels. Finally, we have applied the performances expressions to the Vehicular B and Indoor A environment models. This performances constitute upper bounds for real situations because of the inaccuracy in channel estimation and in synchronization operation.

From the Vehicular B model, frequency selective, we have first illustrated the benefit of the joint detection as compared to the simple desired waveform matched filter, especially when the code margin is little. We have also underlined the clear performance improvement for a coherent reception with 2 or 3 sensors. In this selective channel, the multisensor reception permits to decrease the interference and to facilitate the inversion performed by the equalizer.

From the Indoor A model, weakly selective but also very poor in temporal diversity (non resolved paths), we notice a spectacular improvement of performances due to the diversity gain of the multi-sensor, decreasing the range of amplitude fading.

So, the multi-sensor reception on the mobile is always recommended to efficiently fight against the two different types of distortion introduced by the channel: frequency selectivity and fading process.

\section{References}

[1] Van etten (W.): Maximum likelihood receiver for multiple channel transmission systems. IEEE Transactions on Communications, pp. 276-283, (February 1976).

[2] Turin (G.L.): Introduction to spread-spectrum antimultipath techniques and their application to urban digital radio. Proceedings of the IEEE, vol 68, $n^{0} 3$, pp. 328-353, (March 1980).

[3] Pickholtz (R.L.), Schilling (D.L.), Milstein (L.B.): Theory of spread-spectrum communications - A tutorial. IEEE Transactions on Communications, vol.com-30, $n^{0} 5$, pp. 855-884, (May 1982).

[4] Jung (P.), Blanz (J.): Joint detection with coherent receiver antenna diversity in CDMA mobile radio systems. IEEE Transactions on Vehicular Technology, vol 44, $n^{0} 1$, pp. 76-88, (February 1995).

[5] Madhow (U.), Honig (M.L.): MMSE interference suppression for direct sequence spread-spectrum CDMA. IEEE Transactions on Communications, vol 42, $n^{0} 12$, pp. 31783188, (December 1994).

[6] Wang (X.), Poor (H.V.): Blind multiuser detection: a subspace approach. IEEE Transactions on Information Theory, vol 44, $n^{0}$ 2, pp. 677-690, (March 1998).

[7] Balaban (P.), Salz (J.): Optimum diversity combining and equalization in digital data transmission with applications to cellular mobile radio - Part1: Theorical considerations. IEEE Transactions on Communications, vol 40, $n^{0} 5$, pp. 885-894, (May 1992).

[8] Bourennane (S.), Faure (B.), Lacoume (J.L.): Traitement d'antenne pour des sources bande large. Annales des Télécommunications, vol 45, $n^{0} 7-8, \quad p p$. 384-392, (July 1990).

[9] Diouris (J.F.), Zeidler (J.), Buljore (S.): Spacepath diversity in CDMA using a compact array. Annals of Telecommunications, vol 53, $n^{0} 11-12$, pp. 425-434, (1998).

[10] Buljore (S.), Diouris (J.F.), Saillard (J.): Étude théorique d'un égaliseur multi-capteur associé au critère EQM pour le canal radiomobile (modèle GSM). GRETSI, Juan-les-Pins, pp. 557-560, (September 1995).

[11] Vila (P.), Pipon (F.), Pirez (D.), Fety (L.): Filtrage adapté multidimensioonel pour l'égalisation d'un canal sélectif en fréquence et brouillé. GRETSI, Juan-les-Pins, pp. 553-556, (September 1995).

[12] Pigeonnat (Y.): Alternative solutions for joint detection in TD/CDMA multiple access scheme for UMTS. SPAWC Anapolis, pp. 329-332, (May 1999).

[13] Ros (L.), Jourdain (G.), Arndt (M.): Multi-sensor reception for TDD-UMTS mobile terminal in indoor and pedestrian environments. 11th IEEE International Symposium on PIMRC, London, pp. 1221-1225, (September 2000).

[14] Ros (L.): Réception multi-capteur pour un terminal mobile TDD-UMTS. Report LIS $n^{0} 8 / 2000$, (May 2000).

[15] Capellano (V.): Apport des traitements spatiotemporels pour les transmissions numériques en présence de trajets multiples. Application aux communications acoustiques sous-marines. Phd thesis, INPGrenoble, (May 1998).

[16] Verdu (S.): Multiuser detection. Cambridge university press, (1998).

[17] Proakis (J.G.): Digital communications. McGraw-Hill, third edition, (1995).

[18] JAKES (W.C.): Microwave mobile communications. IEEE PRESS, New-York, (1974).

[19] Marcos (S.) et all: Les méthodes à haute résolution, traitement d'antenne et analyse spectrale. Collection Traitement du Signal, Hermes, (1998).

[20] ETSI : chapter B.1.4.2: Channel impulse response model. UMTS 30.03, TR101-112 V3.2.0, (April 1998).

[21] 3GPP : Technical specification: spreading and modulation (TDD). TS25.223, V3.2.0, (March 2000). 